\title{
EPIPHYSIAL INFARCTION IN A GROWING LONG BONE
}

\author{
An Experimental Study in the Rabbit
}

\author{
M. H. Young, Edinburgh, Scotland
}

From the Department of Pathology, University of Sheffield, England

A growing epiphysis contains a nucleus of bone which enlarges by endochondral ossification of adjacent cartilage. Epiphysial blood vessels are intimately concerned in this process, whereby avascular cartilage is destroyed and replaced by vascular bone. Interruption of the epiphysial blood supply is generally believed to be responsible for Perthes' disease of the femoral head and a number of similar disorders, collectively designated "osteochondritis." Radiology is the principal investigation employed in the diagnosis of these diseases, and histological material is rarely available for study (Gall and Bennett 1942; Haythorn 1949). Consequently, pathological knowledge is incomplete.

The purpose of the experiments described in this paper has been to produce infarction of a growing epiphysis and to study the resulting growth disturbance both radiologically and histologically. The epiphysis studied was that of the rabbit metatarsal, which was selected because it is accessible and may readily be infarcted. The experimental lesion does not bear close comparison with human disease, principally because in order to infarct the metatarsal epiphysis it was necessary also to damage severely or to destroy the related metatarso-phalangeal joint. The results are presented for the information they provide on the function of the blood supply to a growing epiphysis.

\section{EXPERIMENTAL PLAN AND METHODS}

Each rabbit metatarsal has a single epiphysis at the distal end. Most of the surface of the epiphysis is covered with articular cartilage, but this covering is not continuous with the periphery of the growth cartilage, being separated from it by the attachment of the joint capsule to the epiphysial bone. The capsule is thickened on each side of the epiphysis where it forms a collateral ligament. The epiphysis gains its blood supply by vessels which enter it through the attachment of the joint capsule.

Operations were performed on twenty growing rabbits of 390 to 680 grammes weight. These were divided into three groups.

Group 1 (eleven animals)-General anaesthesia was produced with ether and the third right metatarsal bone was exposed by an incision over its dorsum. The metatarso-phalangeal joint was opened by dividing the extensor tendon and collateral ligaments, and the metatarsal epiphysis was exposed. With a scalpel, the attachment of the joint capsule was stripped back off the epiphysis to the level of the growth cartilage, clearly seen at the surface of the bone. The lesion produced by this operation is illustrated in Figure 1. The digit was now amputated through the metatarso-phalangeal joint and the skin wound was sutured. One of these animals died three days after operation and was discarded. The remaining ten were killed one, two, two, three, four, four, five, seven, ten and thirty-four days after operation.

Group 2 (seven animals) - The capsular attachment was stripped back off the third right metatarsal epiphysis, as in group 1, but the digit was not amputated. It was replaced, though the joint capsule and extensor tendon were not repaired, and then the skin wound was sutured. It had been found in the animals of group 1 that changes developed in the articular cartilage which might have been caused either by infarction of the underlying bone or by destruction of the joint with attendant loss of synovial fluid. It was hoped that by leaving the digit in situ, 
although epiphysial infarction would be produced, the joint would not be destroyed and the cause of the changes in the articular cartilage would be made clear. These seven animals were killed four, nine, fifteen, fifteen, twenty-two, twenty-eight and thirty-five days after operation. Group 3 (two animals) - In these animals the third right metatarsal was subjected to capsular stripping and the digit was amputated as in the animals of group 1. Each animal was injected twenty-four hours before death with carrier-free ${ }^{35} \mathrm{SO}_{4}$ (obtained from the Radiochemical Centre, Amersham) diluted in water. The sulphate was injected intraperitoneally in a dose of 2 millicuries per kilogram body weight. Precautions were taken to prevent contamination of the surroundings by excreta. The animals were killed on the same day, one at eight and the other five days after operation.

All animals were killed by intraperitoneal injection of Nembutal and the distal half of the third metatarsal bone was removed from both hind limbs and fixed in 4 per cent formaldehyde.

Radiographs were taken of all specimens after removal and in some cases at intervals during life.

Most bones were decalcified in ethylenediamine tetraacetic acid. Longitudinal sections $5 \mu$ thick were taken at intervals through each block. Sections were stained routinely with haematoxylin and eosin, and in some cases with alcian blue, with toluidine blue, and by the periodic acid-Schiff (PAS) method for mucopolysaccharides.

In three animals, killed at four, nine and fifteen days, both right and left metatarsals were sectioned without prior decalcification and stained by von Kossa's method for calcium salts.

The specimens from the two animals given radioactive sulphate before death were decalcified and studied by routine histology and also by autoradiographs prepared by the stripping film technique (Doniach and Pelc 1950).

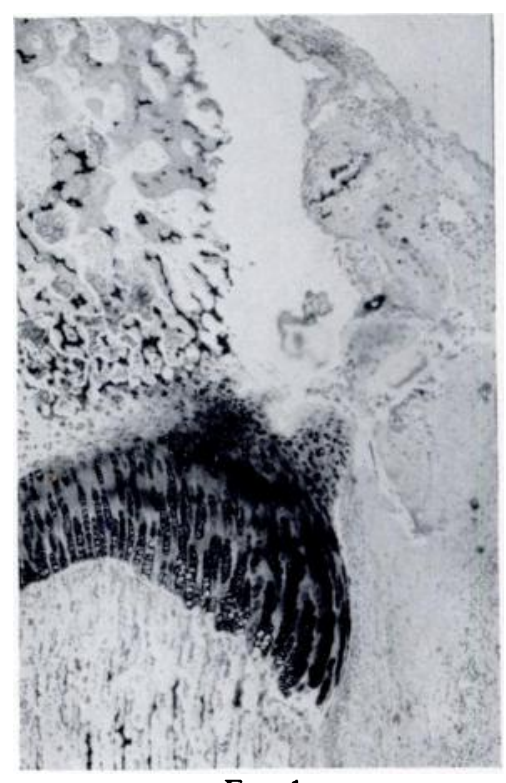

Fig. 1

Metatarsal growth plate three days after operation. The edge of the growth cartilage intervenes between metaphysis below and infarcted epiphysis above. Reflected capsule on right. (Haematoxylin and eosin, $\times 22$.)

\section{RESULTS}

The operation successfully produced infarction of the epiphysis while leaving the blood supply of the metaphysis intact. No differences were observed between those animals in which the digit had been amputated and those in which it was allowed to remain in situ. Proliferation of the growth cartilage was slowed or stopped, and cartilage destruction on its metaphysial face continued, resulting in thinning of the cartilage. Later the epiphysis became revascularised and the growth cartilage became more normal in thickness and structure. The changes occurring in the epiphysis, the growth plate, the metaphysis and the articular cartilage will be described separately.

Changes in the epiphysis--The operation consistently produced necrosis of the entire bony epiphysis. The appearances were those of infarction.

A layer of osteoblasts is present on the surfaces of all bone trabeculae in the normal epiphysis (Fig. 2) but one day after the operation the osteoblasts have disappeared (Fig. 3). This change occurs throughout the epiphysis. Necrosis of epiphysial marrow is also seen twenty-four hours after operation. In contrast, osteocytes in formed bone stain normally for the first few days after operation but in animals killed after more than one week most of the lacunae are empty and the others contain only pyknotic nuclei. The early changes seen in the epiphysis after this operation are the same as those which occur in the metaphysis of the rabbit metatarsal when it is infarcted (Young 1963).

VOL. 48 B, NO. 4, NOVEMBER 1966 
Revascularisation of the epiphysis occurs during the second week after operation. It takes place in two ways. Some blood vessels grow into the bone through the area where the joint capsule was attached; other vessels grow into the epiphysis from the metaphysis, by penetrating through the growth cartilage in a way to be described later.

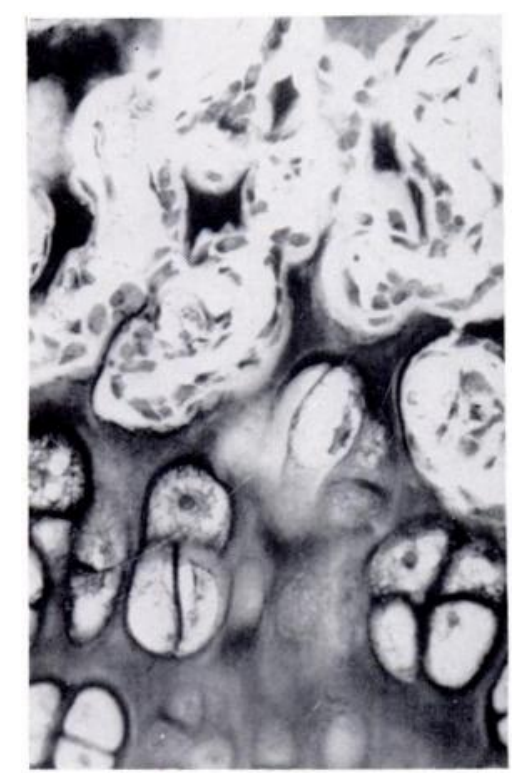

Fig. 2

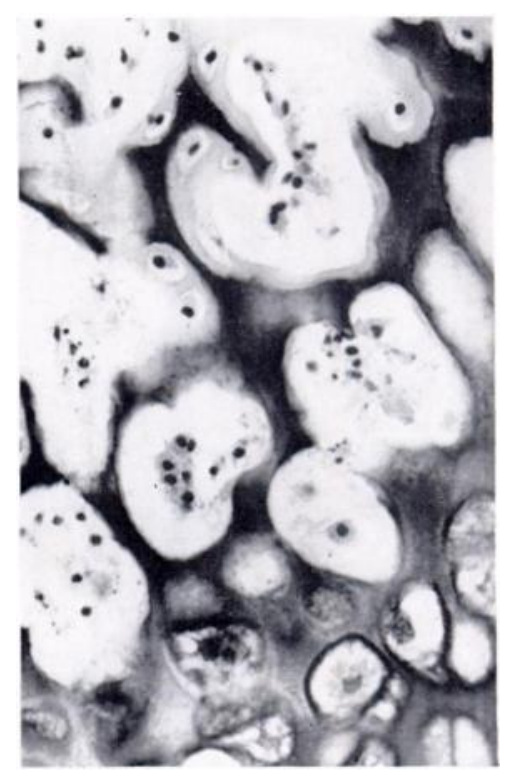

Fig. 3

Figure 2-Normal epiphysis at junction with growth cartilage below. Healthy osteoblasts. (Haematoxylin and eosin, $\times 240$.) Figure 3 -Infarcted epiphysis at junction with growth cartilage, below, one day after operation. Osteoblasts have disappeared. Osteocyte nuclei are still stainable. Compare with Figure 2. (Haematoxylin and eosin, $\times 240$.)

Revascularisation occurs rapidly. No vessels are seen to enter the epiphysis in any specimen from an animal killed during the first seven days after operation, but in all specimens

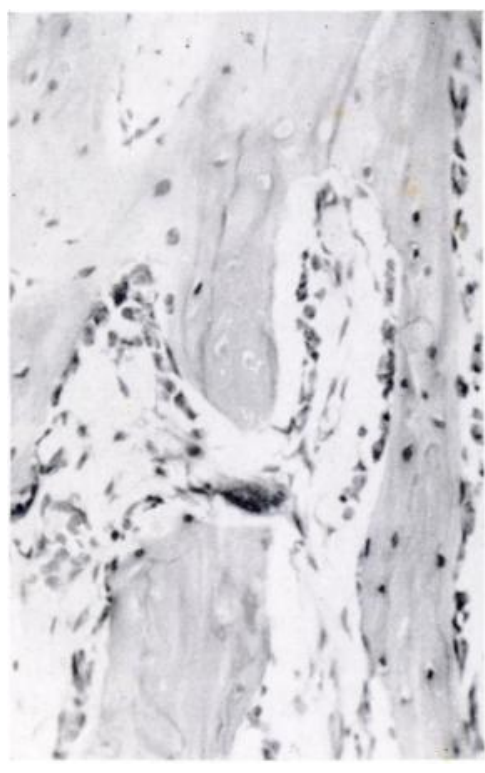

FIG. 4 from animals killed after fifteen or more days newly formed blood vessels are seen throughout the epiphysis. These specimens also contain trabeculae of dead bone, with empty lacunae throughout the epiphysis, which shows that infarction has occurred.

Coincident with revascularisation, new bone is formed within the epiphysis. Layers of new bone are laid down upon the dead trabeculae (Fig. 4). Osteoclasts are present on parts of the surfaces of the dead trabeculae and are presumably engaged in bone absorption. The fate of the infarcted trabeculae is precisely that described by many authors as occurring in bone grafts (Maatz, Lentz and Graf 1954; Ray and Holloway 1957; Young 1964; and others.)

FIG. 4

Epiphysis of rabbit metatarsal twenty-two days after epiphysial infarction. Trabeculae of dead bone with empty lacunae; new bone being laid down in apposition. (Haematoxylin and eosin, $\therefore 140$.) 
The incorporation of dead trabeculae within newly formed bone may be contrasted with what occurs in the infarcted metaphysis when it becomes revascularised. In the metaphysis no new bone is formed on the dead trabeculae (Young 1963).

During the early stages of epiphysial revascularisation bone formation exceeds bone

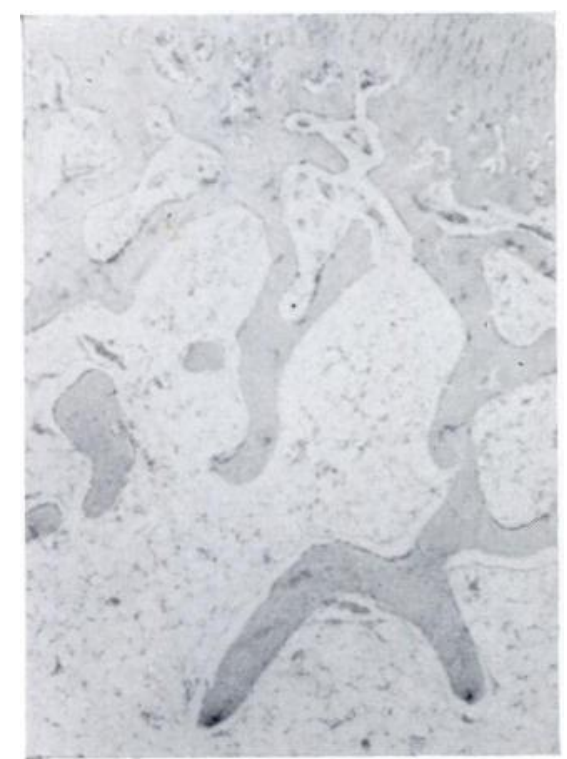

FIG. 5

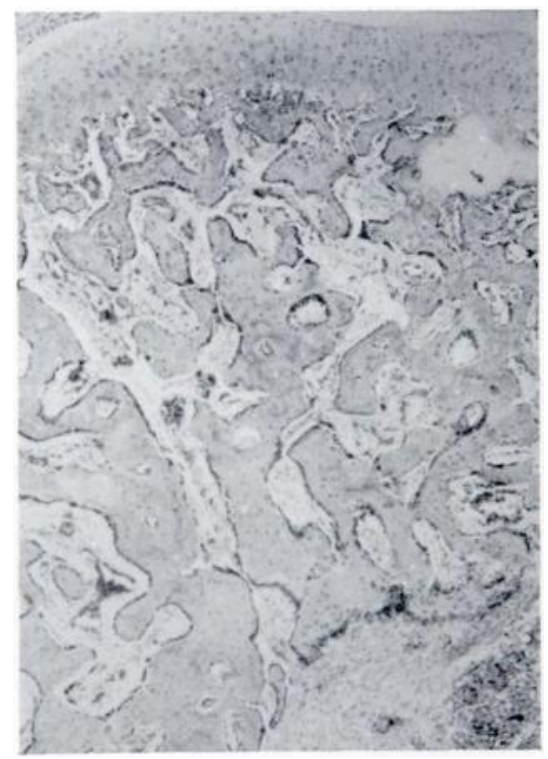

FIG. 6

Figure 5-Normal epiphysis of rabbit metatarsal, to show trabecular pattern. (Haematoxylin and eosin, $\times 30$.) Figure 6-Epiphysis of rabbit metatarsal twenty-two days after epiphysial infarction. Epiphysis now revascularised and much new bone formed. Compare Figure 5. (Haematoxylin and eosin, $\times 30$.)

destruction and more bone than normal is present within the epiphysis (compare Figs. 5 and 6). This change is shown on radiographs as an increased density of the epiphysis, apparent by the eleventh day after operation (Fig. 7). The increased density, once it appeared, persisted until the animal was killed.

In the early stages of revascularisation, myelofibrosis is noticeable. This is less in the later stages when the marrow becomes more cellular.

Changes in the growth cartilage-Endochondral ossification at the metaphysial side of the growth cartilage continues for a time after epiphysial infarction, but the destroyed cartilage cells are not replaced in the normal way, so that the cartilage becomes reduced in thickness (Fig. 8).

To estimate the approximate rate of cell destruction, counts were made of the number of cells in the

FiG. 7

Radiograph eleven days after infarction of third right metatarsal. Revascularisation has occurred and the epiphysis has increased in density due to new bone formation. (The metatarsal has a distal epiphysis. The proximal phalanx has a proximal, flattened epiphysis.) $(\times 2$.)

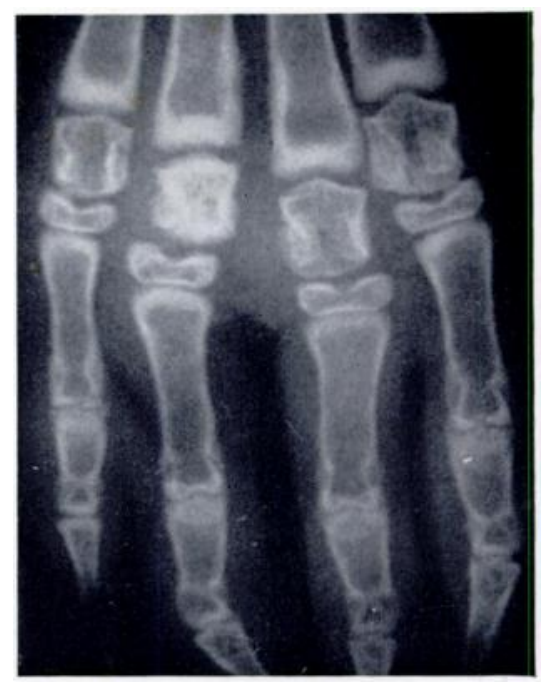

Fig. 7

VOL. $48 \mathrm{~B}$, NO. 4, NOVEMBER 1966 
cartilage columns. Five sections were selected from each test specimen and on each section a count was made of the number of cells in five cartilage columns near the centre of the growth plate and the average number of cells in one growth column calculated. In the same way the average number of cells in one growth column

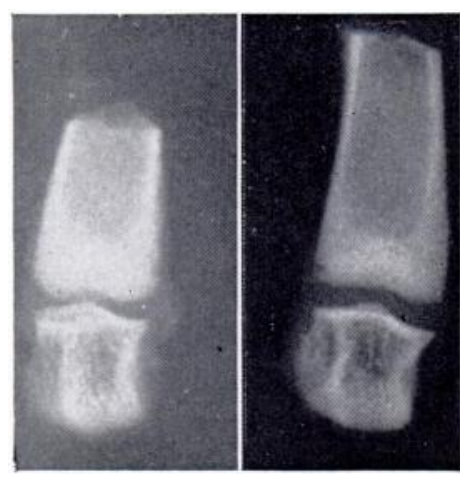

FIG. 8

Left-Radiograph of third metatarsal two days after epiphysial infarction. Right-Untreated metatarsal from same animal. Operated specimen shows reduction in thick ness of translucent band which represents growth cartilage. $(\times 3$. chondrocytes also ceases and so the advancing blood vessels from the metaphysis reach the proliferative zone of the growth plate and it is here that cartilage destruction stops (Figs. 9 and 10). The proliferative zone remains of about normal thickness and is not destroyed. The cartilage columns retain their normal orientation. Later, as will be described, the epiphysis becomes revascularised and the growth plate regains its normal thickness and appearance.

TABLE I

Mean Number of Chondrocytes per Cartilage Column IN THE Third Metatarsal of Four RabBits

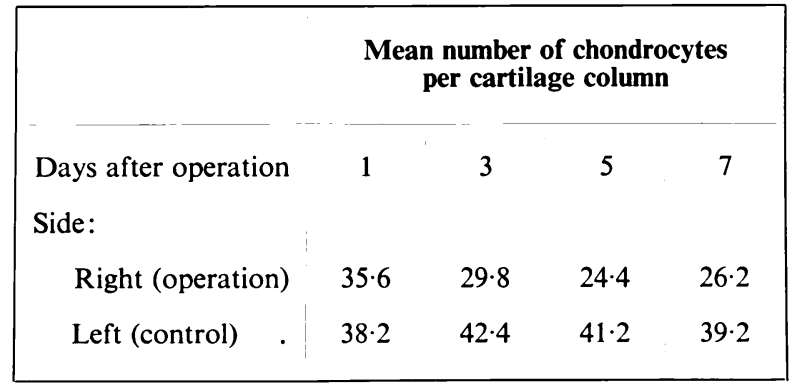

Since proliferation of the growth cartilage is seen to be dependent on an intact epiphysial blood supply it might be expected that death of the entire growth plate would result from epiphysial infarction. This did not occur in any of the experimental animals, but changes were observed which would probably have led to it had the epiphysis not become revascularised. These changes were observed throughout the undifferentiated or first zone of the growth plate and also in localised areas of the second or proliferative zone.

After twenty-four hours the matrix throughout the first zone stains less intensely than the remainder of the growth plate. This change is more marked at two days and at three days it has lost its basophilic and metachromatic staining properties and is slightly eosinophilic 
(Fig. 10). The eosinophilia increases and is intense at seven days. In the specimens from animals killed fifteen or more days after operation, in which the epiphysis is revascularised, the normal basophilia of this zone is restored. These changes in the matrix may be due to a depletion of or change in its mucopolysaccharide content. Autoradiographs of normal growth plates show only slight uptake of sulphate by the cells of zone 1 compared with the rest of the growth plate. This appearance of zone 1 is unchanged five and eight days after epiphysial infarction.

The cells of zone 1 appear to survive until the epiphysis is revascularised, but their morphology changes. Coincident with the increasing eosinophilia of the matrix, the cells

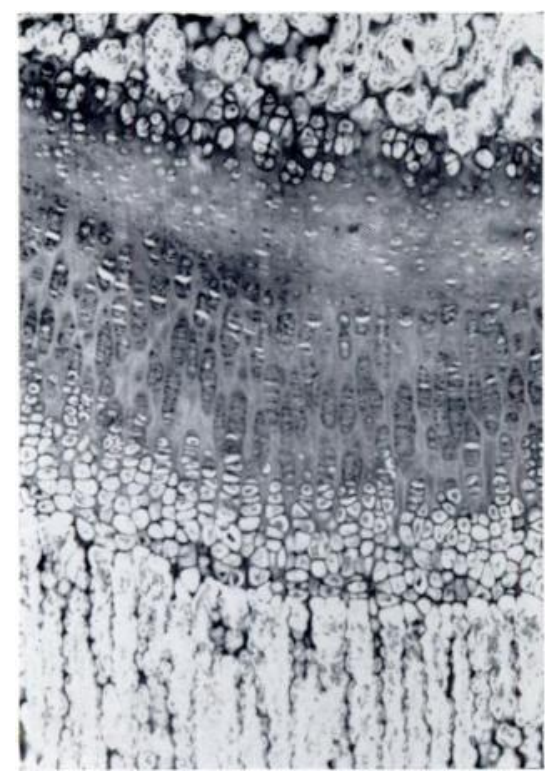

Fig. 9

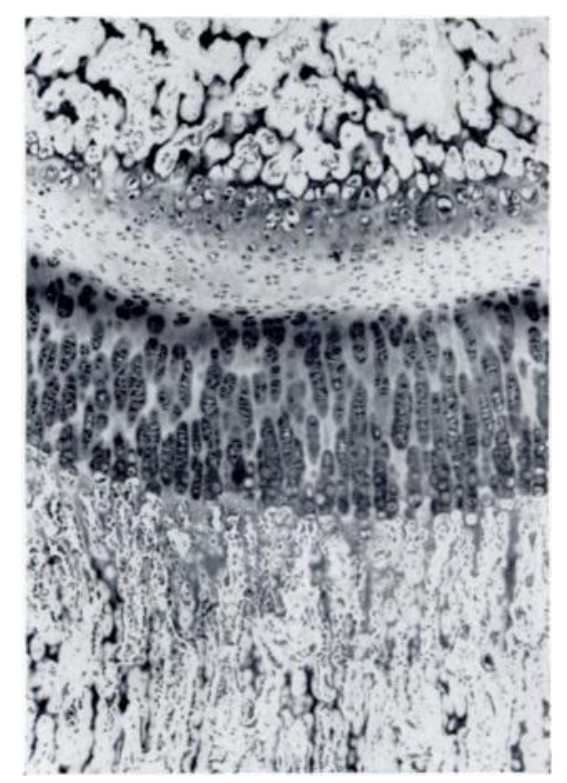

FIG. 10

Figure 9-Normal growth plate of rabbit metatarsal. Epiphysis above, metaphysis below. (Haematoxylin and eosin, $\times 65$.) Figure 10 - Growth plate of rabbit metatarsal three days after epiphysial infarction. Infarcted epiphysis above, metaphysis below. Growth plate reduced in thickness due to destruction of hypertrophied chondrocytes. Pale staining first zone. Thickened trabeculae in metaphysis. Compare with Figure 9. (Haematoxylin and eosin, $\times 65$.)

become fusiform and assume the shape of fibroblasts, with their long axes lying transversely across the growth plate (Fig. 11). Normal cellular morphology is seen in later specimens which show revascularisation of the epiphysis.

The greater part of the proliferative or second zone has a normal histological appearance throughout the duration of the experiment. The cells stop proliferating and since they are not destroyed and do not mature in the usual way the zone remains normal in thickness. Both cells and matrix stain normally throughout the experiment.

Though the greater part of zone 2 remains normal in appearance, around the whole growth plate, in a localised area near its edge, certain changes take place which lead eventually to revascularisation of the epiphysis by vessels passing through the cartilage from the metaphysis. The site of these changes is remarkably constant and appears in longitudinal sections at two areas, one on either side, near but not at the edge of the cartilage. It may be noted here that the growth cartilage of the rabbit metatarsal is not flat but on its epiphysial surface presents a central concavity surrounded by a convex rim. The cartilage changes to be described are first seen in the upper part of zone 2 at the apex of this peripheral rim.

The changes involve both cells and matrix. The cells die; the cytoplasm retracts and does not fill the lacunae; the nuclei become pyknotic; and autoradiographs show a failure to utilise 
${ }^{35} \mathrm{SO}_{4}$. The matrix retains its basophilia and metachromasia but stains less intensely. It appears to consist of a fine network of interlacing fibrils whose principal direction is in the same direction as the cartilage cell columns. These fibrils stain with alcian blue and stain metachromatically with toluidine blue.

These changes are seen first two days after operation. They are present in both the two-day specimens and in every specimen from animals killed at three, four, five and seven days.

At first these areas of cartilage death are small and confined to the upper part of zone 2 . adjacent to zone 1 . They increase in size and come to form two wedge-shaped areas extending

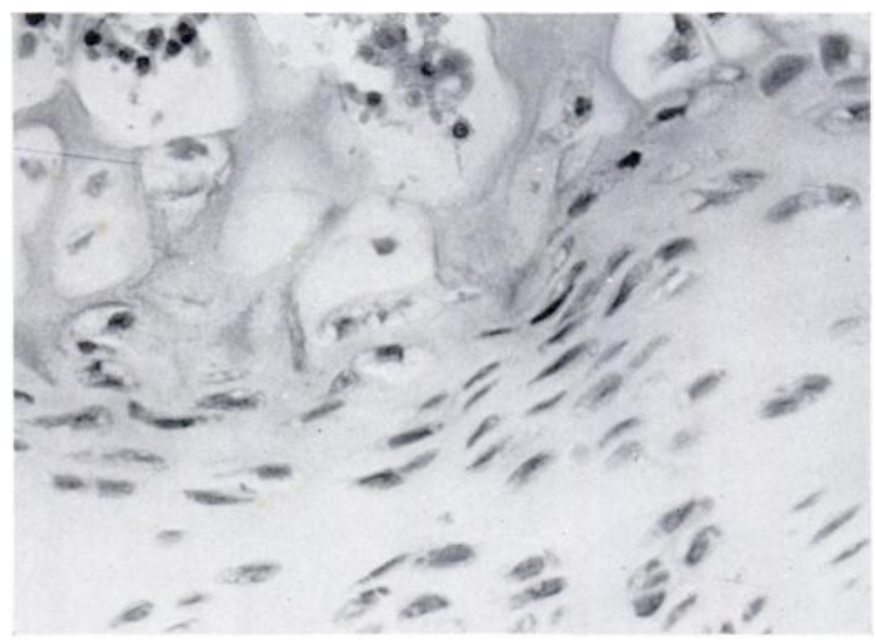

Fig. 11

Undifferentiated zone of growth plate seven days after infarction of epiphysis, above. Fibroblast-like cells. (Haematoxylin and eosin, $\times 300$.)

through the whole of zone 2, with their apices towards the metaphysis (Figs. 12 and 13). This area of cartilage death spares the main central part of the growth plate in every specimen. Sometimes it spreads peripherally to the edge of the growth plate (Fig. 14), or it may leave a region of living cartilage at the edge of the growth plate.

By five days after operation the zones of cartilage death have extended through to reach the metaphysial side of the growth plate (Fig. 15) and at this time blood vessels are beginning to grow from the metaphysis into the cartilage towards these areas of dead tissue (Fig. 16).

These vascular ingrowths have a different pattern from those normally seen in the metaphysis. Instead of destroying hypertrophied cartilage cells and growing up into the cell columns these vessels grow into the matrix between the cell columns. The chondrocytes nearest the vascular processes do not hypertrophy but remain small, with compact cytoplasm. The normal columnar arrangement of cartilage cells is preserved.

At seven days vascular invasion has progressed further and at one side of the growth plate the dead cartilage has been destroyed and a vascular communication has become established between metaphysis and epiphysis (Fig. 17). This break in the cartilage is lined by bone and separates off a small peripheral fragment of cartilage from the main part of the growth cartilage. The vascular ingrowths are localised and the central part of the growth plate remains intact.

The vascular breaks in the growth cartilage appear to persist and when the epiphysis becomes revascularised a completely normal growth cartilage is reformed from the central part of the previous one (Fig. 18). The peripheral rim of cartilage may also survive and when it does so it is seen in longitudinal sections as a fragment of cartilage displaced towards the 


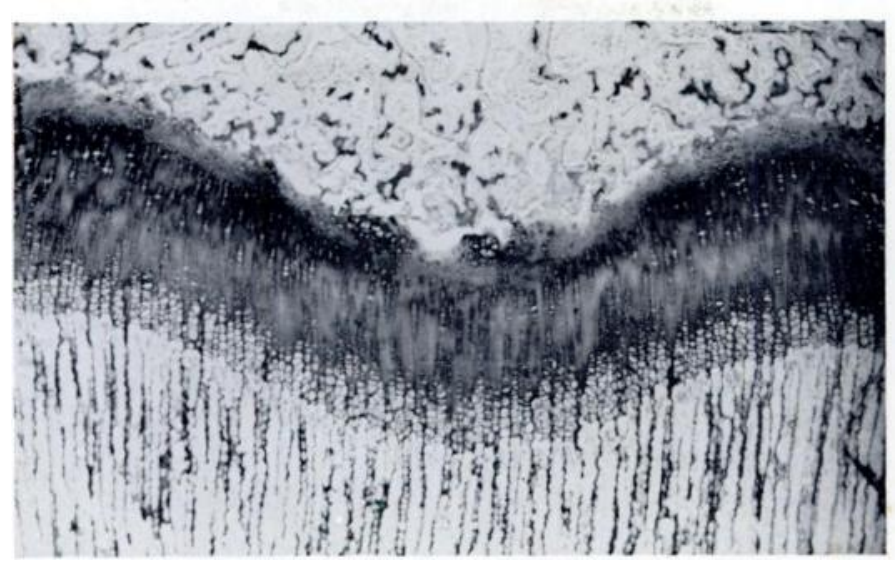

Fig. 12

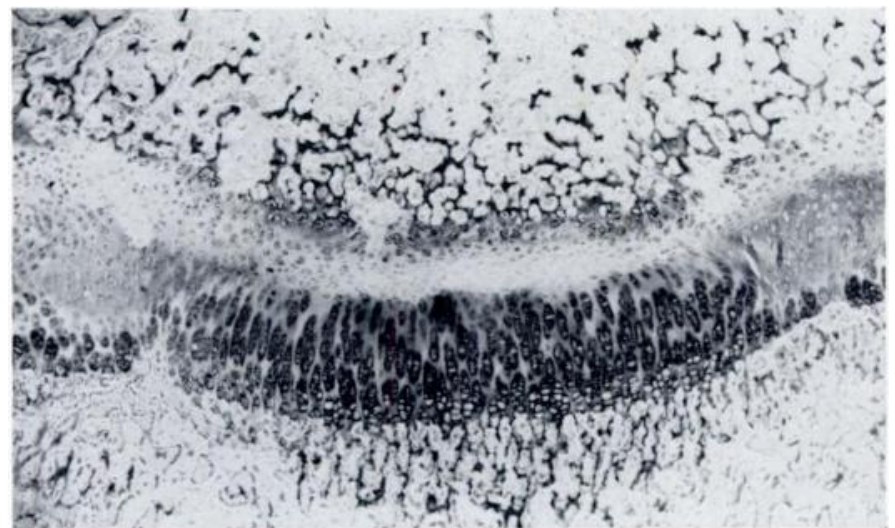

Fig. 13

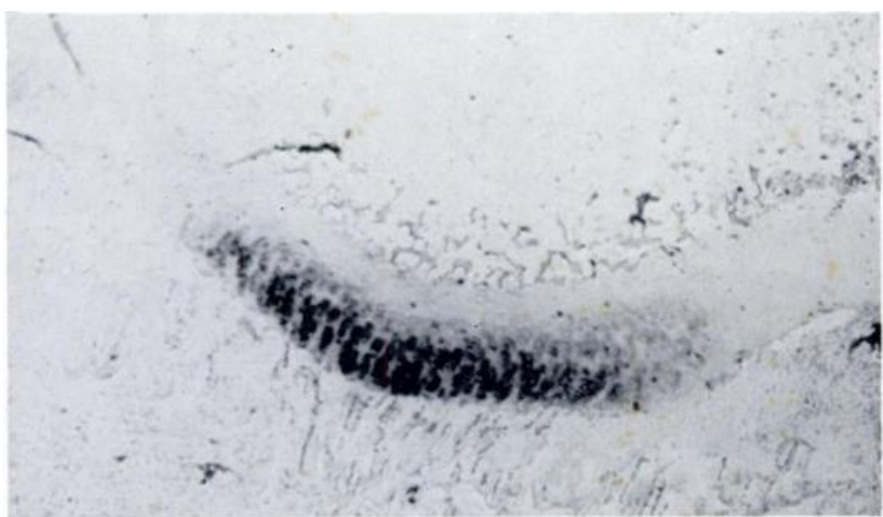

FIG. 14

Figure 12-Normal growth plate. Same animal as shown in Figure 13. (Haematoxylin and eosin, $\times 24$.) Figure 13 -Growth plate five days after epiphysial infarction. Infarcted epiphysis above, metaphysis below. Wedge of dead cartilage near each side of cartilage. Compare with Figure 12. (Haematoxylin and eosin, $\times 24$.) Figure 14 -Autoradiograph of growth plate five days after epiphysial infarction. Normal uptake of sulphate by cells in centre part of plate. Depressed uptake on each side. Infarcted epiphysis above, metaphysis below. $(\times 24$. $)$ 


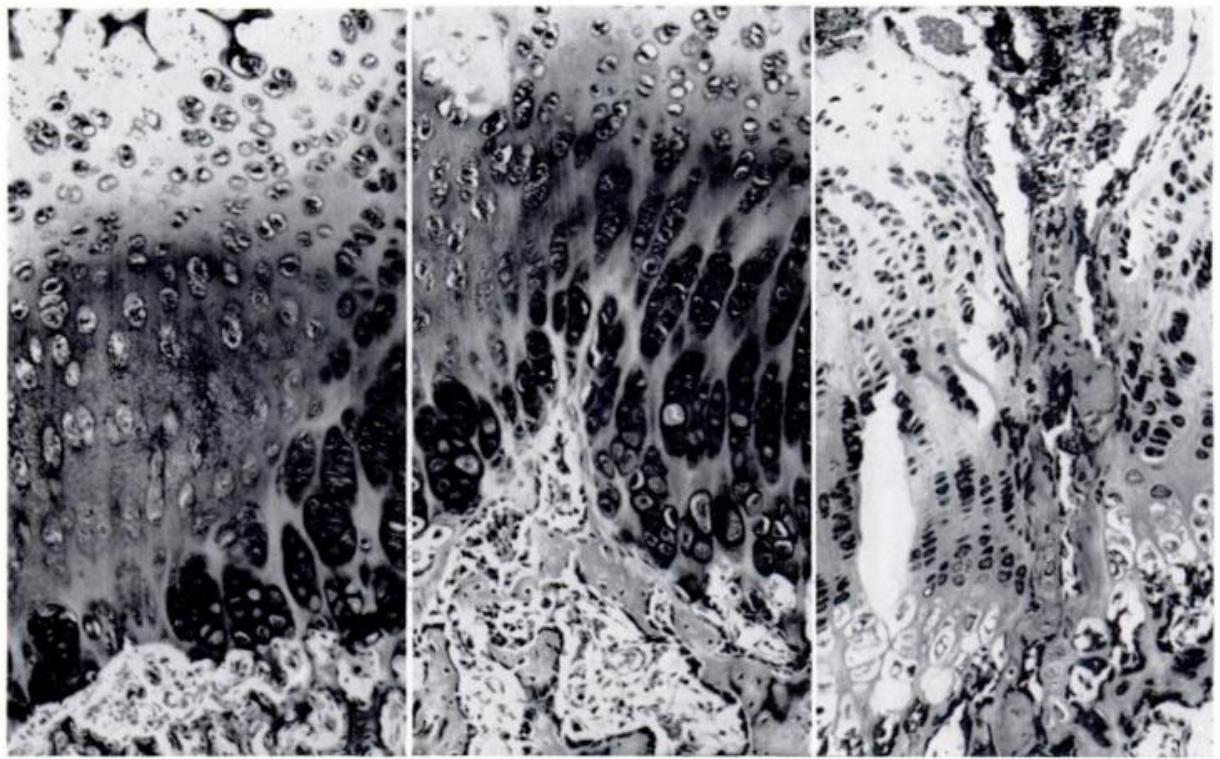

FIG. 15

Fig. 17

Figure 15-Growth plate five days after epiphysial infarction. A wedge of dead cartilage extends from infarcted epiphysis above to metaphysis below. Near margin of growth plate. (Haematoxylin and eosin, $\times 80$.) Figure 16 -Peripheral part of growth cartilage five days after epiphysial infarction. Blood vessels from the metaphysis below are growing into the cartilage towards zone of dead cartilage above. (Haematoxylin and eosin, $\times 80$.) Figure 17-Peripheral part of growth cartilage seven days after epiphysial infarction. A vascular channel lined by bone has grown through from the metaphysis below to the infarcted epiphysis above. (Haematoxylin and eosin, $\times 80$.)

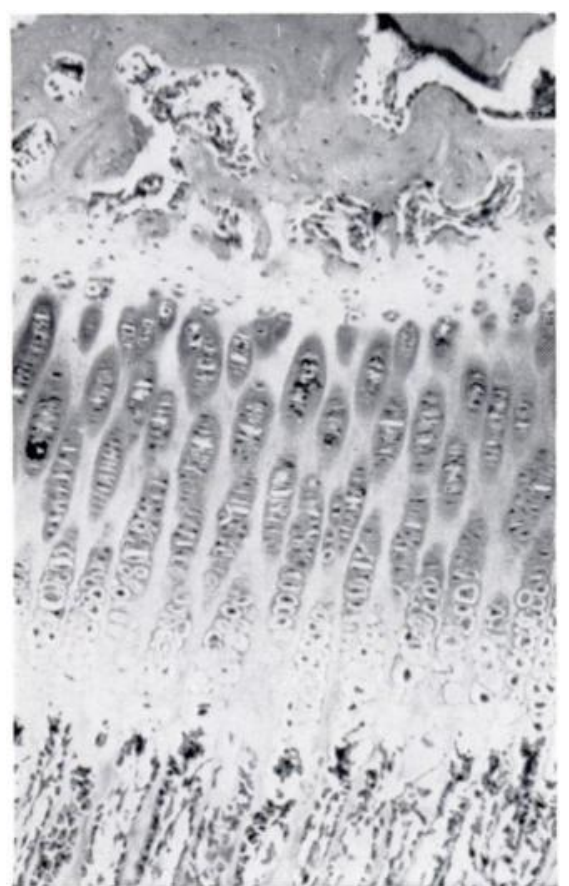

FIG. 18

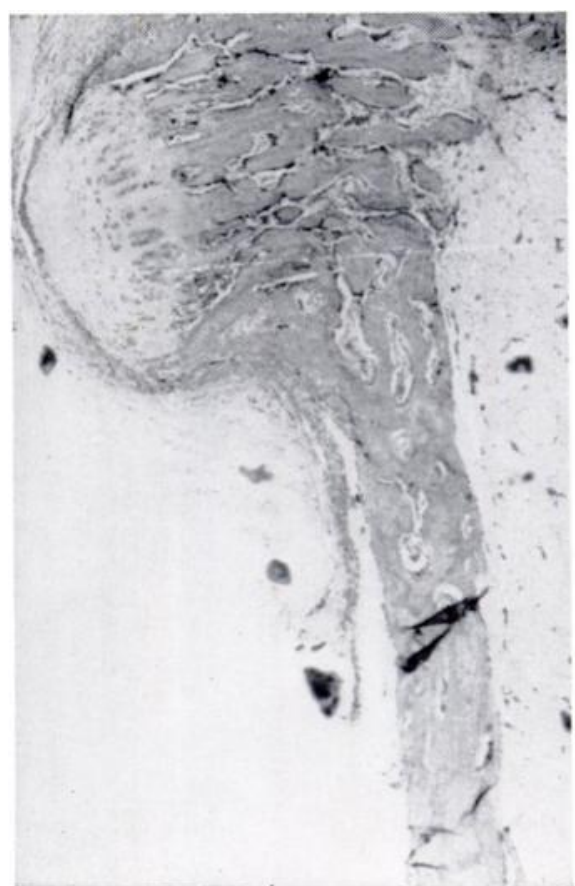

Fig. 19

Figure 18-Normal structure of growth plate twenty-eight days after epiphysial infarction. Revascularised epiphysis above. Metaphysis below. (Haematoxylin and eosin, $\times 65$.)

Figure 19-Displaced fragment of cartilage on surface of metatarsal thirty-four days after epiphysial infarction. The growth plate lies above. (Haematoxylin and eosin, $\times 23$.) 
shaft and showing endochondral ossification, with its cells tending to retain a columnar arrangement (Fig. 19).

Changes in the metaphysis - In no specimen does the metaphysis show any evidence of interference with its blood supply. One day after operation the histological appearance of the metaphysis is entirely normal (compare Figs. 20 and 21).

Changes are present in both the specimens examined two days after operation. The trabeculae are of normal length but thicker than normal. However, at the base of the trabeculae, at their attachment to the growth cartilage, they are thinner than normal and some have lost their connection with the cartilage. This change is more pronounced at three days, when blood vessels have grown across between the growth cartilage and the metaphysis and many

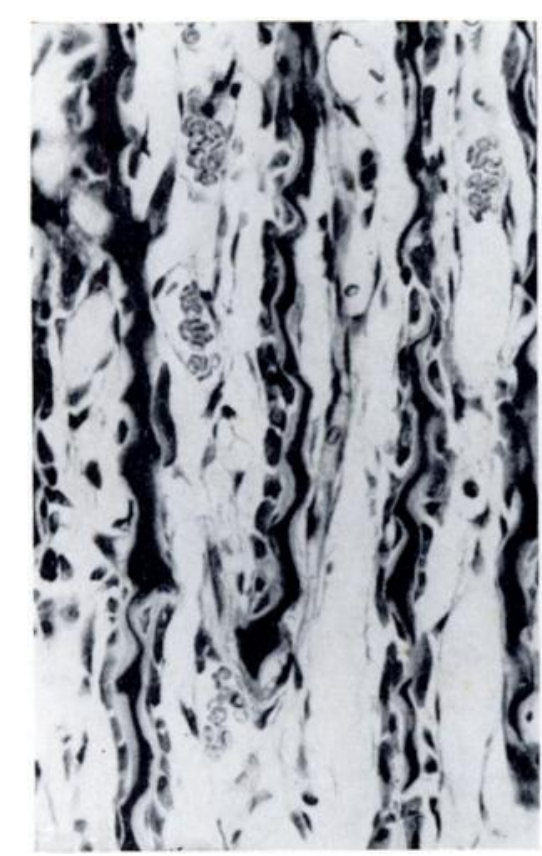

FIG. 20

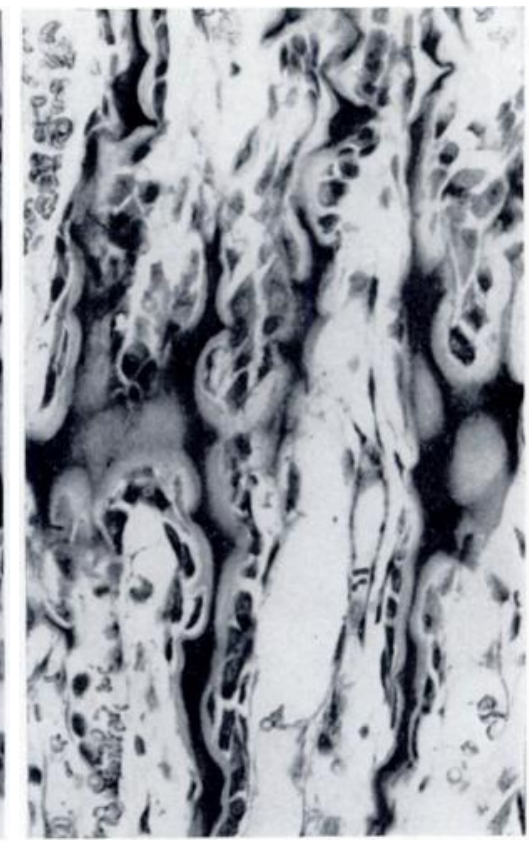

Fig. 21

Figure 20-Normal metaphysis. Osteoblasts in apposition to metaphysial trabeculae. (Haematoxylin and eosin, $\times 240$.) Figure 21 -Metaphysis, one day after epiphysial infarction. Healthy osteoblasts. Compare with Figure 20 (from same section as Figure 3). (Haematoxylin and eosin, $\times 240$.)

of the trabeculae appear to have been nipped off at their bases (Fig. 22). It is best developed below the areas of cartilage where the wedge-shaped areas of cartilage death develop, but is seen across the whole width of the metaphysis.

At four days the changes already noted are present and in addition the metaphysial trabeculae are shorter than normal. This change is apparent radiologically (Fig. 23) and histological sections show the depth of the metaphysis to be only about twice that of the growth cartilage. The normal metaphysis is about three times as thick as the growth cartilage and undergoes continuous absorption at its diaphysial surface, where osteoclasts may be seen at the ends of the trabeculae. The shortening observed at four days appears to be due to this absorption continuing at a time when endochondral ossification of the growth cartilage is slowing down.

By seven days after operation the metaphysis is further narrowed, being only about the same thickness as the growth cartilage itself (Fig. 24). There is gross disturbance of the trabecular pattern, the trabeculae being thick and interwoven, resembling the pattern seen in the normal epiphysis.

Vol. 48 B, No. 4, NOVEMBER 1966 
At ten days the trabecular pattern and width of the metaphysis is similar to that at seven days, and in addition bone has formed in apposition to the cartilage, forming an incomplete layer over its metaphysial surface (Fig. 25). This bony walling off of the growth cartilage is not present in specimens from animals killed more than ten days after operation. In these later specimens revascularisation of the epiphysial side of the growth plate is established, and coincident with this and probably because of it, the trabecular pattern in the metaphysis becomes histologically normal.

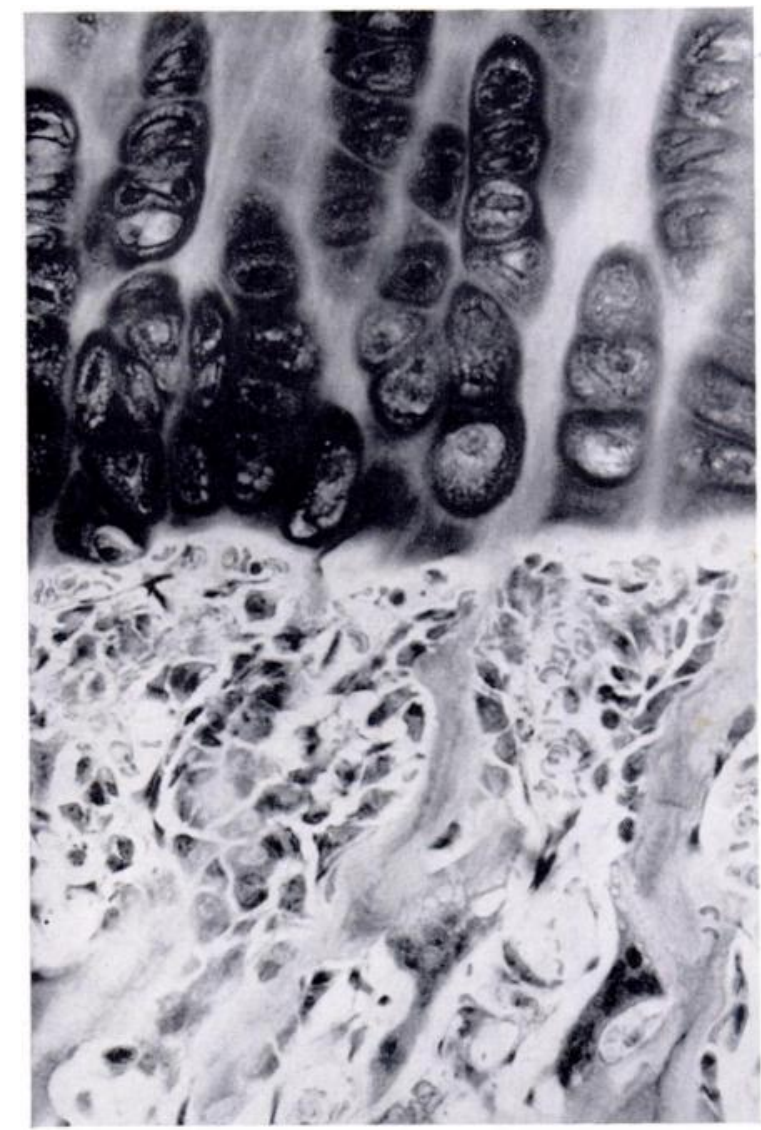

FIG. 22

Metaphysial side of growth plate three days after epiphysial infarction. The hypertrophied layer of chondrocytes has been destroyed. Healthy osteoblasts present in metaphysis

below. Some trabeculae nipped off at attachment to cartilage. (Haematoxylin and eosin, $\times 310$.)

Changes in the articular cartilage-Degenerative changes developed in the articular cartilage after operation and these were the same in both the animals subjected to capsular stripping with amputation of the digit and the animals in which capsular stripping was performed, but the digit was not amputated. In the latter group the metatarso-phalangeal joint was unstable and the proximal phalanx of the digit tended to become drawn proximally, below the epiphysis of the metatarsal. A normal joint was not preserved in any animal.

One day after operation the articular cartilage appears histologically normal. At two days the matrix has lost its normal basophilia and metachromasia and become eosinophilic. Eosinophilia of the matrix persisted for the thirty-five days that the experiment continued.

The cells of the articular cartilage stain normally at first, but at four days some cells nearest the bone have compact basophilic cytoplasm and eosinophilic nuclei, features which 
may indicate cell death. These cells do not persist once revascularisation has occurred. The remaining cells of the articular cartilage stain normally throughout the duration of the experiment.

The articular cartilage remains of normal thickness until fifteen days after operation, but in the four specimens from animals killed later than this it is reduced to about one-third to one-half the normal thickness. In these four specimens the articular cartilage has become covered with a fibrous pannus, a feature not present in earlier specimens.

\section{DISCUSSION}

Epiphysial infarction in the rabbit metatarsal has been found to produce profound changes in the underlying growth cartilage. It prevents the proliferation and normal maturation of chondrocytes and also produces localised areas of cartilage death. These changes may now be compared with the findings of other workers.

Trueta and his colleagues (Trueta and Morgan 1960; Trueta and Little 1960) described in detail the structure of the blood vessels related to the epiphysial side of the growth cartilage and described an experiment designed to study their function (Trueta and Amato 1960). They studied the proximal tibial
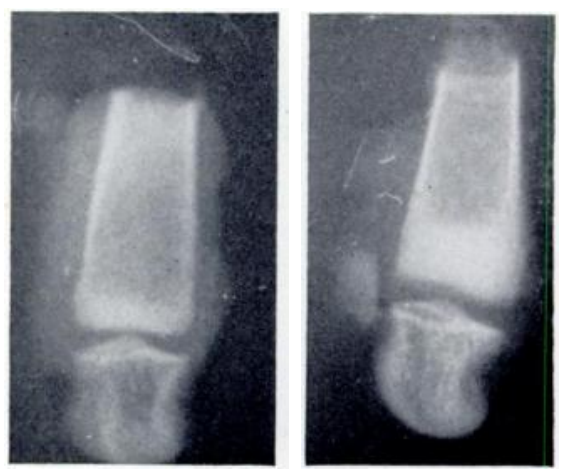

Fig. 23

Left-Radiograph of third metatarsal four days after epiphysial infarction. RightRadiograph of untreated metatarsal from same animal. Operated specimen shows narrowing of both growth cartilage and metaphysis. $(\times 3$.)

growth cartilage of growing rabbits after drilling a small hole on the medial side of the epiphysis and packing this with polythene film to prevent revascularisation. This operation was considered to produce a localised area of ischaemia overlying the growth plate. Trueta and Amato found that around these areas epiphysial bone trabeculae died, as shown by empty lacunae, seen as early as two days after operation. This is in contrast to the present experiment, where empty lacunae were not seen until one week after operation. In the growth cartilage variable changes were seen. Sometimes a localised transient widening occurred and sometimes a segment of the growth cartilage died beneath the infarcted area of epiphysis. The authors concluded that the epiphysial vessels sustained the life and proliferation of the growth cartilage and that vessels in the metaphysis were solely concerned in the calcification and destruction of this structure.

Brashear (1963) described changes in the growth cartilage resulting from experimentally produced infarction of the distal femoral epiphysis in growing rats. The knee joint was disarticulated and the limb amputated at this level and then a cuff of periosteum about two millimetres wide was removed from the circumference of the femur one to two millimetres proximal to the growth plate. This operation was found to produce abnormalities in all the epiphyses studied and infarction was usually massive, affecting most if not all of the epiphysis. It was implied that the blood supply to the metaphysis was unimpaired though the histology of the metaphysis in the early days after operation was not described.

The findings in the rabbit metatarsal after epiphysial infarction closely resemble the changes described by Brashear in the rat femoral epiphysis. Thinning of the growth plate due to loss of the hypertrophied cell layer occurred in both cases, showing that both proliferation and maturation of chondrocytes were depressed or arrested. In both experiments the metaphysial trabeculae became shorter, thicker and more interwoven, and in both the trabeculae tended to become nipped off at their attachment to the cartilage. Walling off of the metaphysial side of the growth cartilage by bone was described by Brashear and has also been found to occur in the rabbit. In the rat, however, this process appears to have been more complete. All these changes occurred at approximately the same rate in both rabbit and rat.

Vol. 48 B, No. 4, NOVEMBER 1966 
In both the rat and the rabbit revascularisation of the epiphysis occurred by metaphysial vessels growing through the growth cartilage. Vascular communications between metaphysis and epiphysis were noted after one week by Brashear and have been found to develop at this time in the rabbit. A remarkable feature in the rabbit is that these vascular connections consistently occur in the same localised part of the growth plate. The main, central part of

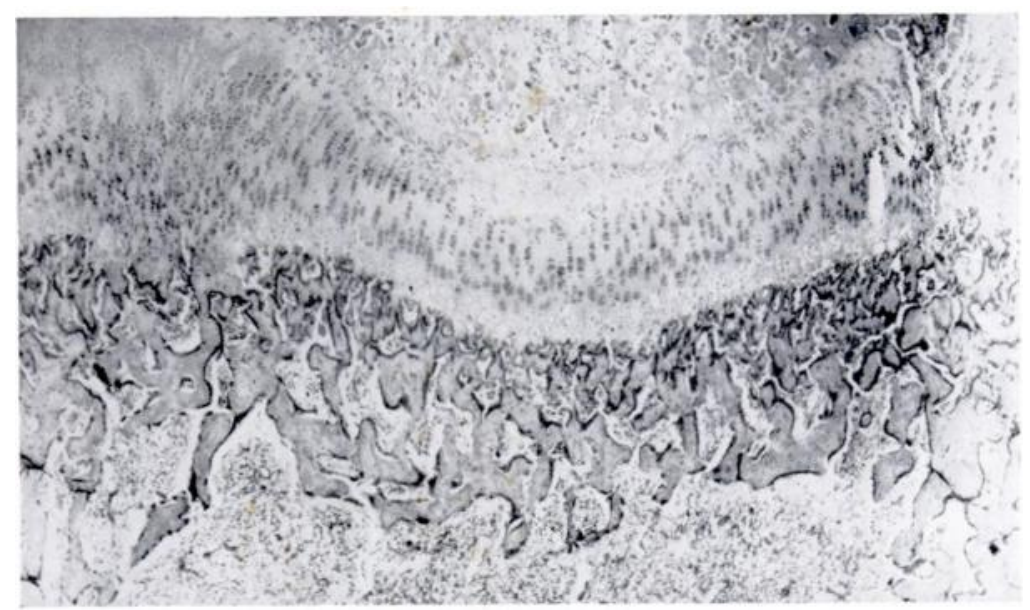

Fig. 24

Growth plate seven days after epiphysial infarction. Infarcted epiphysis above. The metaphysis, below, is about the same thickness as the growth cartilage itself and the trabeculae are thickened and interwoven. (Haematoxylin and eosin, $\times 24$.)

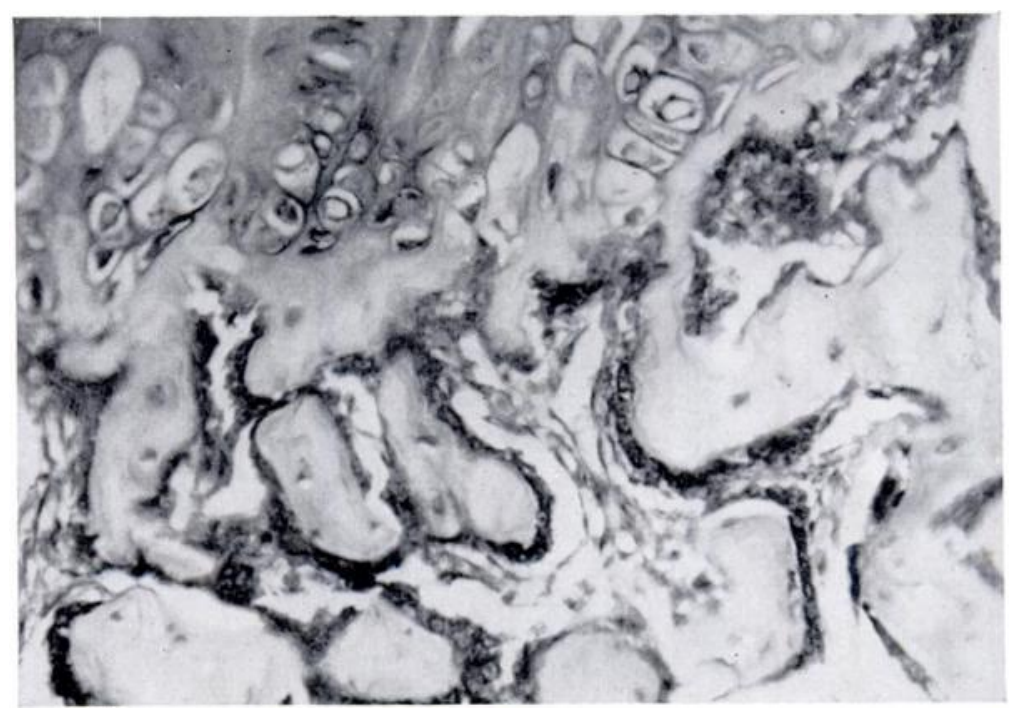

Fig. 25

Metaphysial side of growth cartilage ten days after epiphysial infarction. Bone, below, has formed in apposition to the cartilage above. (Haematoxylin and eosin, $\times 190$.)

the growth plate is not seen to be penetrated by blood vessels in any animal. In contrast, Brashear found vascular communications to develop throughout the growth plate. This diffuse vascular invasion sometimes leads to destruction of large parts of the growth cartilage.

Growth in length of the rat femur was depressed but did not appear to be affected in the rabbit metatarsal. The rabbit third metatarsal is normally intermediate in length between 
the second and fourth metatarsals. This relationship in length was preserved in all the animals studied (Fig. 7) and may be attributed to early epiphysial revascularisation together with the preservation intact of the main central part of the growth plate.

The differences which have been observed between the findings of Brashear and those of the experiments described here may be species differences between rats and rabbits but are more likely to be due to a difference in the lesion produced by the two experiments. Removal of a cuff of periosteum in the region of the metaphysis, though it may infarct only the epiphysis, may also disturb the normal flow of blood through the metaphysis. Though accounts of vascular anatomy differ in detail it is well established that many connections exist between the blood vessels within the metaphysis and those in the overlying periosteum (Lewis 1956; Brookes 1958; Morgan 1959; Nelson, Kelly, Peterson and Janes 1960; Brookes, Elkin, Harrison and Heald 1961). Whichever direction of flow predominates in the distal metaphysis of the rat femur, it is likely that the experiment described by Brashear did alter metaphysial blood flow in addition to interrupting epiphysial blood supply. In the present experiment no histological changes are present in the metaphysis at a time when the epiphysis is clearly infarcted, and it is likely that the changes are caused by epiphysial infarction alone.

It is apparent that the orientation of the cartilage cell columns is very stable and that the growth plate is able to survive and regenerate after severe interference with its normal nutrition.

The areas of cell death which follow epiphysial infarction are remarkably localised, always appearing in the same part of the growth plate. Vascular penetration of the growth plate occurs only through these areas of dead cartilage. This is in contrast to the changes after metaphysial infarction (Young 1963) where the growth plate becomes perforated throughout its substance by vessels which grow through from the epiphysis to revascularise the metaphysis.

Narrowing of the growth cartilage, shortening and thickening of the metaphysial trabeculae and walling off of the metaphysial surface of the growth cartilage by bone have been shown to occur in the proximal tibial epiphysis of the rat during the normal process of ageing (Becks, Simpson and Evans 1945a) and also after hypophysectomy (Becks, Simpson and Evans 1945b). These similarities suggest that epiphysial ischaemia may play a part in the normal age changes, but this has not been demonstrated.

The increase in radiographic density which occurs as a result of revascularisation and new bone formation within the infarcted epiphysis has been previously described by Bobechko and Harris (1960) and by Brashear (1963). New bone formation in the human femoral head has been described in Perthes' disease (Haythorn 1949), and it has been pointed out (Jacobs 1960) that radiological changes may not be present in the early stages of this condition. These observations suggest that the increased radiological density which occurs in Perthes' disease and in the past has been considered to result from bone necrosis, may really be due to revascularisation of an infarcted epiphysis and new bone formation within it. Brashear (1963) found that increased radiographic density of the infarcted rat femoral epiphysis persisted until the beginning of the fifth week, but that after that time osteoporosis developed. Osteoporosis was not noted in the experiments described in this paper, but might have developed if the animals had been studied for a longer period.

The articular cartilage changes observed in these experiments are difficult to interpret because the metatarso-phalangeal joint was destroyed or damaged.

\section{SUMMARY}

1. Experiments are described in which total infarction of the epiphysis was produced in the metatarsal bones of growing rabbits.

2. After operation both proliferation and normal maturation of the cells of the growth plate were slowed or stopped. Cartilage destruction on the metaphysial side of the growth cartilage continued with consequent thinning of the cartilage. Localised areas of cell death appeared 
in the growth cartilage as early as the second day after operation. These increased in size and led to revascularisation of the epiphysis by metaphysial vessels which grew through the growth cartilage, reaching the epiphysis seven days after operation. The main, central part of the growth cartilage survived intact and its normal structure was restored after epiphysial revascularisation took place. Vessels growing into the bone from outside also contributed to revascularisation of the epiphysis. After revascularisation occurred, new bone formation led to increased radiographic density of the epiphysis.

I am grateful to the late Professor D. H. Collins and to Mr F. W. Holdsworth for helpful advice and criticism; to Dr G. Meachim for advice in the use of radioactive sulphate; to Mrs P. M. Glossop and Mrs S. Williams for the preparation of histological sections; and to $\mathrm{Mr} \mathrm{T}$. L. Platts for his help with radiology and photomicrographs. The work was supported by a grant from the Empire Rheumatism Council.

\section{REFERENCES}

Becks, H., Simpson, M. E., and Evans, H. M. (1945a): Ossification at the Proximal Tibial Epiphysis in the Rat. I. Changes in Females with Increasing Age. Anatomical Record, 92, 109.

Becks, H., Simpson, M.E., and Evans, H. M. (1945b): Ossification at the Proximal Tibial Epiphysis in the Rat. II. Changes in Females at Progressively Longer Intervals following Hypophysectomy. Anatomical Record, 92, 121.

Bobechko, W. P., and Harris, W. R. (1960): The Radiographic Density of Avascular Bone. Journal of Bone and Joint Surgery, 42-B, 626.

Brashear, H. R., Jun. (1963): Epiphyseal Avascular Necrosis and Its Relation to Longitudinal Bone Growth. Journal of Bone and Joint Surgery, 45-A, 1423.

Brookes, M. (1958): The Vascularization of Long Bones in the Human Foetus. Journal of Anatomy, 92, 261.

Brookes, M., Elkin, A. C., Harrison, R. G., and Heald, C. B. (1961): A New Concept of Capillary Circulation In Bone Cortex. Some Clinical Applications. Lancet, 1, 1078.

Doniach, I., and Pelc, S. R. (1950): Autoradiograph Technique. British Journal of Radiology, 23, 184.

Gall, E. A., and Bennetr, G. A. (1942): Osteochondritis Deformans of the Hip (Legg-Perthes Disease) and Renal Osteitis Fibrosa Cystica. Report of a Case with Anatomic Studies. Archives of Pathology, 33, 866.

HAYThorn, S. R. (1949): Pathological Changes Found in Material Removed at Operation in Legg-CalvéPerthes Disease. Journal of Bone and Joint Surgery, 31-A, 599.

JACOBS, B. W. (1960): Early Recognition of Osteochondrosis of Capital Epiphysis of Femur. Journal of the American Medical Association, 172, 527.

Lewis, O. J. (1956): The Blood Supply of Developing Long Bones with Special Reference to the Metaphyses. Journal of Bone and Joint Surgery, 38-B, 928.

MaAtz, R., Lentz, W., and Graf, R. (1954): Spongiosa Test of Bone Grafts for Transplantation. Journal of Bone and Joint Surgery, 36-A, 721.

Morgan, J. D. (1959): Blood Supply of Growing Rabbit's Tibia. Journal of Bone and Joint Surgery, 41-B, 185.

Nelson, G. E., Jun., Kelly, P. J., Peterson, L. F. A., and Janes, J. M. (1960): Blood Supply of the Human Tibia. Journal of Bone and Joint Surgery, 42-A, 625.

Ray, R. D., and Holloway, J. A. (1957): Bone Implants. Preliminary Report of an Experimental Study. Journal of Bone and Joint Surgery, 39-A, 1119.

Trueta, J., and Amato, V. P. (1960): The Vascular Contribution to Osteogenesis. III. Changes in the Growth Cartilage Caused by Experimentally Induced Ischaemia. Journal of Bone and Joint Surgery, 42-B, 571.

Trueta, J., and Litrle, K. (1960): The Vascular Contribution to Osteogenesis. II. Studies with the Electron Microscope. Journal of Bone and Joint Surgery, 42-B, 367.

Trueta, J., and Morgan, J. D. (1960): The Vascular Contribution to Osteogenesis. I. Studies by the Injection Method. Journal of Bone and Joint Surgery, 42-B, 97.

YounG, M. H. (1963): Changes in the Growth Cartilage Resulting from Ischaemic Necrosis of the Metaphysis. Journal of Pathology and Bacteriology, 85, 481.

Young, M. H. (1964): The Repair of Experimental Defects in Rabbit Skulls. Observations after Implantation with Decalcified, Deproteinised and Deep Frozen Homogenous Whole Bone. Journal of Bone and Joint Surgery, 46-B, 329. 